\title{
WAVE MOTION NEAR A BREAKWATER ROUNDHEAD
}

\author{
Takahide Honda ${ }^{1}$, Peter Wellens ${ }^{2}$ and Marcel van Gent ${ }^{3}$
}

\begin{abstract}
COMFLOW is a general 3D free-surface flow solver. The numerical method is based on the Navier-Stokes equations in a porous medium, with additional force terms to represent the (turbulent) interaction of the flow with the medium. The free surface is displaced by means of the Volume-Of-Fluid method. The main objective in this paper is to validate the permeable flow model in 3D. Tailor-made physical model tests were performed for this purpose. In the experiment surface elevations are measured inside and around a permeable structure with 18 wave gauges in total. The measurements are represented well by the simulation results.
\end{abstract}

Keywords: VOF; Navier-Stokes; permeable flow; rubble-mound breakwater; physical model tests

\section{INTRODUCTION}

Validation of numerical models for waves in 3D is difficult, mainly because of spurious reflection from domain boundaries. We wish to validate ComFLOW (Kleefsman et al., 2005) for 3D wave applications. ComFLOW includes a numerical method that is based on the Navier-Stokes equations. The free surface is advected by means of an improved Volume-of-Fluid algorithm. With the numerical model we aim to simulate wave interaction with 3D coastal structures, similar to physical models in a 3D wave basin.

Consider a relatively large basin such as the Delta Basin at Deltares, see Figure 1. In the basin, spurious reflection of waves that reach the basin boundaries are absorbed by means of spending beaches on all sides of the basin, except on the side with the wave maker. At Deltares, the wave maker in the Delta Basin is equipped with Active Reflection Compensation (ARC) to prevent spurious rereflection of waves back towards the structure.

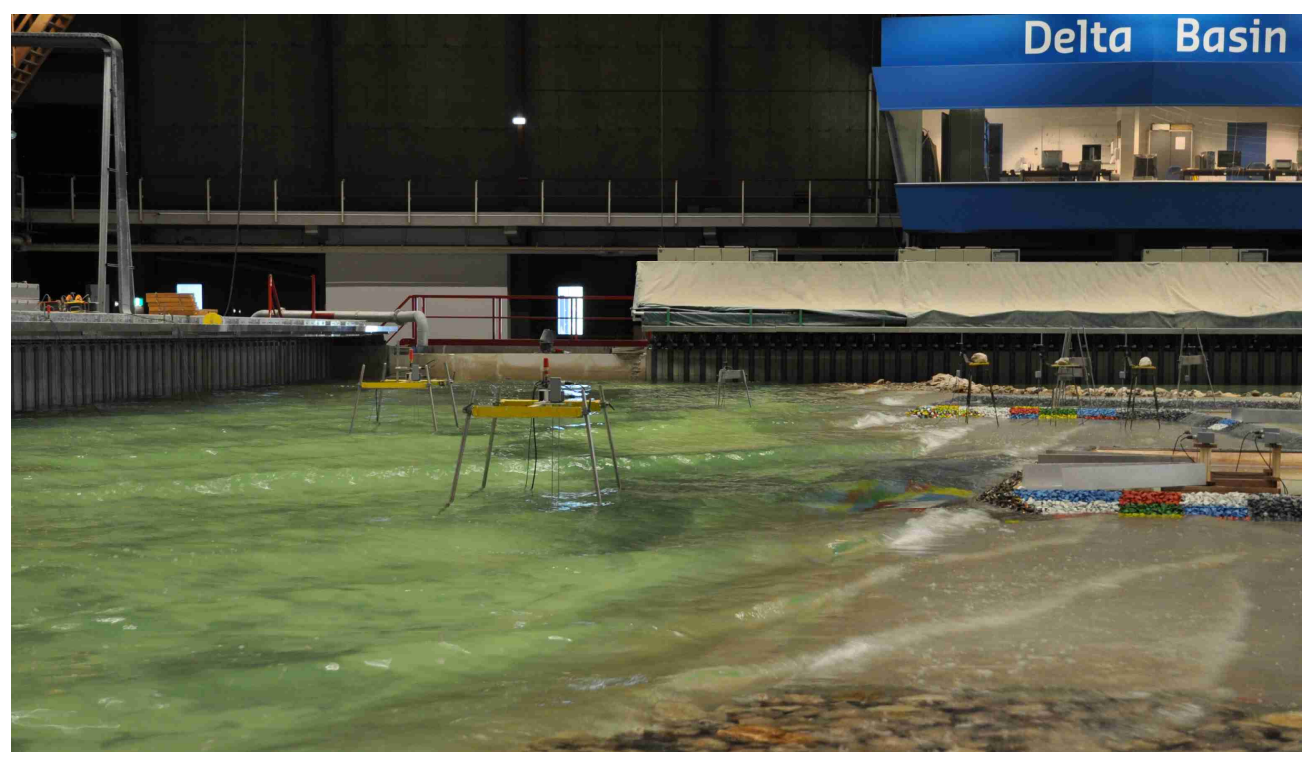

Figure 1. Delta Basin at Deltares

Spending beaches are an appropriate measure in the basin, because there often is sufficient room. Together with ARC, spurious re-reflection of waves will not disturb the experiment. In a numerical domain, however, dissipation zones - the numerical equivalent of spending beaches - are a necessary evil: they take up a considerable amount of the domain and, consequently, of the computer memory and processor effort. In circumstances with limited available computer memory, it becomes a modeling decision how to distribute the available resources in the compromise between resolving the

\footnotetext{
${ }^{1}$ Taisei Corporation, 344-1, Nase-cho, Totsuak-ku, Yokohama 245-0051, Japan; hndtkh01@pub.taisei.co.jp

2 Deltares, P.O. Box 177, 2600 MH Delft, the Netherlands; peter.wellens@deltares.nl

${ }^{3}$ Deltares, P.O. Box 177, 2600 MH Delft, the Netherlands; marcel.vangent@deltares.nl
} 
flow near the structure with sufficient detail, and preventing re-reflection of waves with dissipation zones.

An alternative for dissipation zones is to develop accurate local absorbing boundary conditions (ABCs) for waves. An $\mathrm{ABC}$ for long-crested, dispersive waves with normal incidence to the boundary is presented in Wellens et al. (2009). This ABC, however, gives too much reflection as the wave direction with respect to the outward normal to the boundary becomes larger than approximately $30^{\circ}$. Currently, work is ongoing to extend the $\mathrm{ABC}$ to be accurate for larger wave directions with respect to the outward normal, but the development has not yet finished at the moment of writing.

To validate COMFLOW for $3 \mathrm{D}$ applications, we chose not to devote a significant amount of computational resources to dissipation zones, but rather to the accuracy of the simulation results itself. An experiment was devised that is tailored to the capabilities of the present boundary conditions in ComFLOW. In this experiment, a breakwater roundhead is placed in the Scheldt Flume. There will be significant wave reflection from the side walls of the flume, which is unacceptable in regular experiments. But in our application, the complex wave fields resulting from the interaction with the side walls, are advantageous for the validation of the numerical model.

\section{GOVERNING EQUATIONS}

For flow through permeable media, the Navier-Stokes equations need to account for the permeability of the medium. With COMFLOW, we will not compute the flow through the pores themselves, i.e. the grid resolution in our simulations will be much coarser than the pores of the rock material that we wish to model. Therefore, a volume-averaged method is adopted, in which we assume that the properties of the permeable structure, such as the porosity, are homogenous throughout (part of) the structure.

Besides porosity, we also need to account for the viscous interaction of the flow with the rocks in the permeable structure. We cannot represent all the (turbulent) boundary layers around the individual stones and will therefore model the viscous interaction with an additional volume averaged friction force in the Navier-Stokes equations that depends on the flow velocity. A similar approach is adopted in Darcy's Law, which was adapted by Forchheimer to account for turbulent flows. The friction force is as follows:

$$
R(u)=(\hat{a}+\hat{b}|u|) u
$$

in which $\hat{a}$ is a Darcy-type coefficient, $\hat{b}$ is a Forchheimer-type coefficient and $u$ is the actual flow velocity in the permeable structure and not the bulk velocity. The expressions for $\hat{a}$ and $\hat{b}$ are based on Van Gent (1995). Friction force (1) is part of the extended Navier-Stokes equations for permeable flow.

The extended continuity equation for permeable flow is:

$$
\nabla \cdot(\varepsilon u)=0
$$

in which $\varepsilon$ is the porosity. We assume constant viscosity and an incompressible fluid. Then the momentum equation reads:

$$
\varepsilon \frac{\partial u}{\partial t}+\nabla \cdot(u \varepsilon u)=v \nabla \cdot(\varepsilon \nabla u)-\frac{\varepsilon}{\rho} \nabla p-\varepsilon R(u)+\varepsilon F
$$

Here, $v$ is the viscosity of the fluid, $\rho$ is the density of the fluid, $p$ is the pressure and $F$ is an external force vector (such as, for instance, gravity).

\section{NUMERICAL METHOD}

Eqs. (2) and (3) require discretization for the implementation in COMFLOW. In this section, the discretization of the individual terms in the Navier-Stokes equation will be discussed. These terms will be combined later in the paragraph concerning the time discretization. In COMFLOW, a finite volume discretization has been adopted. To that end, we will now present the weak form of the Navier-Stokes equations. The continuity equation: 


$$
\int_{\Gamma}(\varepsilon u) \cdot n \partial V
$$

Here, $n$ is the normal vector to the boundary of the control volume $\Gamma$. The weak formulation of the momentum equation becomes:

$$
\begin{aligned}
\varepsilon \int_{\Omega} \frac{\partial u}{\partial t} d V+ & \int_{\Gamma} u(u \varepsilon \cdot n) d S= \\
& \quad \int_{\Gamma}(\varepsilon \nabla u) \cdot n d S-\frac{\varepsilon}{\rho} \int_{\Gamma} p n d S-\varepsilon \int_{\Omega} R(u) d V+\varepsilon \int_{\Omega} F d V
\end{aligned}
$$

\section{Space discretization of the continuity equation}

Figure 2 shows a control volume for the continuity equation. The control volume corresponds to a grid cell on which the flow variables are defined. Note that the variables are staggered; velocities are defined at the cell faces, the pressure is defined in the cell centre (not shown in Figure 2).

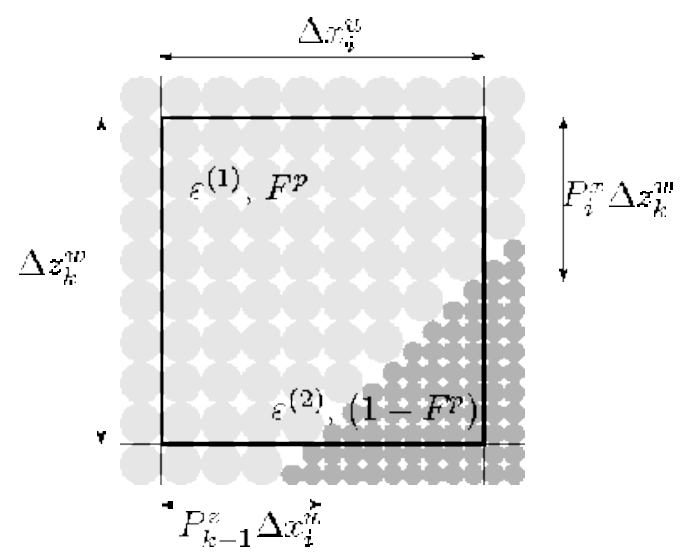

Figure 2. Control volume for the continuity equation.

The control volume in Figure 2 is divided in two regions with different porosity. The grid sizes $\Delta x_{i}^{u}$ and $\Delta z_{k}^{w}$ are scaled by means of the dimensionless coefficients $P_{i}^{x}$ and $P_{k}^{z}$ to represent the outer dimensions of the two regions within the cell. The volumes of the regions are given by $F^{p} \Delta x_{i}^{u} \Delta z_{k}^{w}$ and $\left(1-F^{p}\right) \Delta x_{i}^{u} \Delta z_{k}^{w}$. Note, however, that the volume of the cell that is open for fluid, in which we account for the porosity, is equal to:

$$
F^{b}=\left(\varepsilon^{(1)} F^{p}+\varepsilon^{(2)}\left(1-F^{p}\right)\right) \Delta x_{i}^{u} \Delta z_{k}^{w}
$$

We will determine the flow through the pores of the cell face indicated by $i$ :

$$
\Phi_{i}=u_{i} A_{i}^{x} \Delta z_{k}^{w}
$$

in which $\Phi_{\mathrm{i}}$ represents the flux through the cell face with index $i$ and:

$$
A_{i}^{x}=\varepsilon^{(1)} P_{i}^{x}+\varepsilon^{(2)}\left(1-P_{i}^{x}\right)
$$

is what we call an aperture. It is the area of the cell face that is open to fluid. The discrete equivalent of the continuity equation in Eq. (4) is as follows:

$$
\Phi_{i}-\Phi_{i-1}+\Phi_{k}-\Phi_{k-1}=0
$$


And after substitution of all fluxes $\Phi$ around the circumference of the control volume, the discrete continuity equation reads:

$$
\left(u_{i} A_{i}^{x}-u_{i-1} A_{i-1}^{x}\right) \Delta z_{k}^{w}+\left(w_{k} A_{k}^{z}-w_{k-1} A_{k-1}^{z}\right) \Delta x_{i}^{u}=0
$$

\section{Space discretization of the momentum equation}

\section{Time derivative}

In Figure 3, a control volume for the momentum equation is shown. The control volume is defined in between two consecutive grid cells. In the momentum equation, we require the size of the control volume. It is determined as the weighted average of the grid cell volumes, such as in Figure 4, on either side. First, we determine the volume of the pores in cell with index $i$ :

$$
F_{i}^{b}=\varepsilon^{(1)} F_{i}^{p}+\varepsilon^{(2)}\left(1-F_{i}^{p}\right)
$$

Then we determine the volume of the open region in the cell with index $i+1$ :

$$
F_{i+1}^{b}=\varepsilon^{(1)} F_{i+1}^{p}+\varepsilon^{(2)}\left(1-F_{i+1}^{p}\right)
$$

And finally, we determine the weighted average of the two:

$$
F_{i}^{u}=\frac{F_{i}^{b} \Delta x_{i}^{u}+F_{i+1}^{b} \Delta x_{i+1}^{u}}{\Delta x_{i}^{u}+\Delta x_{i+1}^{u}}
$$

The space discretization of the time derivative of the momentum equation then becomes:

$$
\varepsilon \int_{\Omega} \frac{\partial u}{\partial t} d V \approx F_{i}^{u} \Delta x_{i}^{p} \Delta z_{k}^{w} \frac{\partial u}{\partial t}
$$

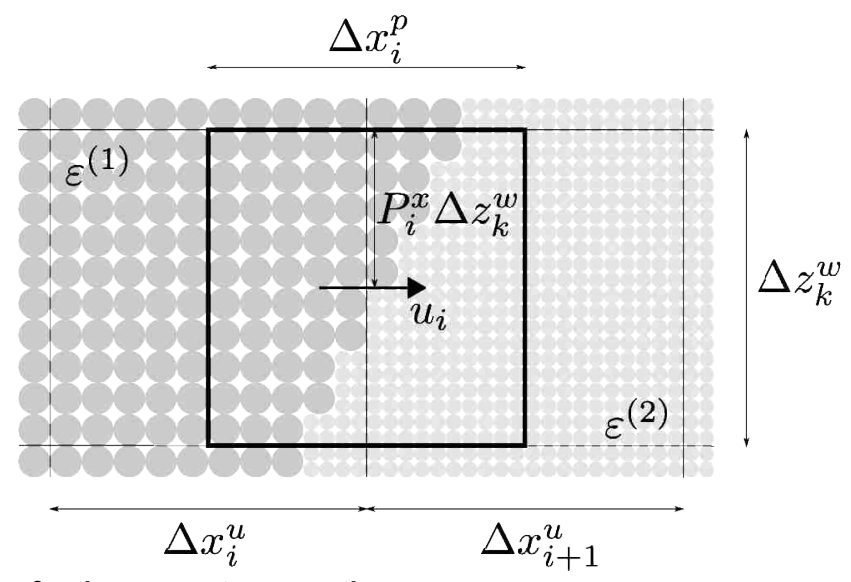

Figure 3. Control volume for the momentum equation.

\section{Convective term}

The Finite Volume discretization of the convective term is equal to the combination of fluxes over the boundary of the control volume:

$$
\int_{\Gamma} u(u \varepsilon \cdot n) d S \approx \Phi_{i+\frac{1}{2}}-\Phi_{i-\frac{1}{2}}+\Phi_{k+\frac{1}{2}}-\Phi_{k-\frac{1}{2}}
$$

in which the fluxes through the right and the top control volume boundary are: 


$$
\begin{aligned}
\Phi_{i+\frac{1}{2}}= & \frac{1}{2}\left(u_{i, k} A_{i}^{x}+u_{i+1, k} A_{i+1}^{x}\right) \Delta z_{k}^{w} \frac{1}{2}\left(u_{i, k}+u_{i+1, k}\right)+ \\
& \frac{1}{2} \xi\left|u_{i, k} A_{i, k}^{x}+u_{i+1, k} A_{i+1, k}^{x}\right| \Delta z_{k}^{w} \frac{1}{2}\left(u_{i, k}-u_{i+1, k}\right) \\
\Phi_{k+\frac{1}{2}}= & \frac{1}{2}\left(w_{i, k} A_{i, k}^{z}+w_{i+1,1} A_{i+1, k}^{z}\right) \Delta x_{i}^{u} \frac{1}{2}\left(u_{i, k}+u_{i, k+1}\right)+ \\
& \frac{1}{2} \xi\left|w_{i, k} A_{i, k}^{z}+w_{i+1, k} A_{i+1, k}^{z}\right| \Delta x_{i}^{u} \frac{1}{2}\left(u_{i, k}-u_{i, k+1}\right)
\end{aligned}
$$

Note in (16) that the parameter $\xi$ determines the amount of upwind that is specified. The apertures $A$ in Eq. (16) can be determined from Figure 3. As an example we will provide:

$$
A_{i}^{x}=\varepsilon^{(1)} P_{i}^{x}+\varepsilon^{(2)}\left(1-P_{i}^{x}\right)
$$

\section{Viscous term}

The discretization of the viscous term in this paper is relatively inaccurate compared to the other terms discussed here because it accounts for the porosity in a simplified manner. There are two reasons why we may assume that this discretization does not influence the results to a great extent. With COMFLOW we are primarily interested in wave loads as a result of steep waves; the type of flow in these waves is dominated by convection. In addition, inside permeable structures, the friction force term is by far dominant over the viscous term. For the discretization of the viscous term, we revert to the volume integral:

$$
v \int_{\Omega} \nabla \cdot \nabla u d V
$$

The divergence of the gradient of $u$ is approximated as follows, see Figure 3:

$$
\nabla \cdot \nabla u \approx \Psi=\frac{1}{\Delta x_{i}^{p}}\left(\frac{u_{i+1, k}-u_{i, k}}{\Delta x_{i+1}^{u}}-\frac{u_{i, k}-u_{i-1, k}}{\Delta x_{i}^{u}}\right)+\frac{1}{\Delta z_{k}^{w}}\left(\frac{u_{i, k+1}-u_{i, k}}{\Delta z_{k}^{p}}-\frac{u_{i, k}-u_{i, k-1}}{\Delta z_{k-1}^{p}}\right)
$$

And Eq. (19) is multiplied by the volume of the momentum cell (see Eq. (13)) to obtain the full discretization of the viscous term:

$$
v \int_{\Omega} \nabla \cdot \nabla u d V \approx v F_{i}^{u} \Delta x_{i}^{p} \Delta z_{k}^{w} \Psi
$$

Pressure and force term

For the pressure term we adopt the following discretization, see Figure 3:

$$
\frac{\varepsilon}{\rho} \int_{\Omega} p n d S \approx \frac{1}{\rho}\left[\left(p_{i+1}-p_{i}\right) \varepsilon^{(1)} P_{i}^{x}+\left(p_{i+1}-p_{i}\right) \varepsilon^{(2)}\left(1-P_{i}^{x}\right)\right] \Delta z_{k}^{w}(21)
$$

For the force term, a discretization is adopted in such a way that in hydrostatic circumstances the discrete form of $1 / \rho \nabla p=F$ is satisfied. The discretization of the force term in $x$-direction then becomes:

$$
\int_{\Omega} F_{x} d V \approx\left[F_{x} \varepsilon^{(1)} P_{i}^{x}+F_{x} \varepsilon^{(2)}\left(1-P_{i}^{x}\right)\right] \Delta x_{i}^{p} \Delta z_{k}^{w}
$$


Friction force term

For the integration of the friction force term over the control volume, we adopt a weighted average method. The friction force in the control volume with index $i$ (see Figure 3) is:

$$
\begin{aligned}
R(u)_{i-\frac{1}{2}}=\frac{1}{\varepsilon^{(1)} F_{i}^{p}+\varepsilon^{(2)}\left(1-F_{i}^{p}\right)}\left[\varepsilon^{(1)}\left(a^{(1)}+b^{(1)} \sqrt{u_{i}^{2}+w_{c}^{2}}\right) F_{i}^{p}+\right. \\
\left.\varepsilon^{(2)}\left(a^{(2)}+b^{(2)} \sqrt{u_{i}^{2}+w_{c}^{2}}\right)\left(1-F_{i}^{p}\right)\right] u_{i}
\end{aligned}
$$

The friction force in the cell with index $i+1$ :

$$
\begin{aligned}
& R(u)_{i+\frac{1}{2}}=\frac{1}{\varepsilon^{(1)} F_{i+1}^{p}+\varepsilon^{(2)}\left(1-F_{i+1}^{p}\right)} {\left[\varepsilon^{(1)}\left(a^{(1)}+b^{(1)} \sqrt{u_{i}^{2}+w_{c}^{2}}\right) F_{i+1}^{p}+\right.} \\
&\left.\varepsilon^{(2)}\left(a^{(2)}+b^{(2)} \sqrt{u_{i}^{2}+w_{c}^{2}}\right)\left(1-F_{i+1}^{p}\right)\right] u_{i}
\end{aligned}
$$

in which $w_{\mathrm{c}}$ is approximated as the simple average of the vertical velocities at the position of $u_{\mathrm{i}}$ :

$$
w_{c}=\frac{1}{4}\left(w_{i, k}+w_{i, k-1}+w_{i+1, k}+w_{i+1, k-1}\right)
$$

Then, the total discretization of the friction force term becomes:

$$
\int_{\Omega} R(u) d V \approx F_{i}^{u} \Delta x_{i}^{p} \Delta z_{k}^{w} \frac{R_{i-\frac{1}{2}} \Delta x_{i}^{u}+R_{i+\frac{1}{2}} \Delta x_{i+1}^{u}}{\Delta x_{i}^{u}+\Delta x_{i+1}^{u}}
$$

\section{Time discretization of the momentum equation}

The space discretization of the continuity equation and the momentum equation can be written in matrix form and combined with a Forward Euler time integration:

$$
\begin{aligned}
& M_{\varepsilon} \mathbf{u}^{n+1}=0 \\
& \Omega_{\varepsilon} \frac{\mathbf{u}^{n+1}-\mathbf{u}^{n}}{\Delta t}=-C_{\varepsilon}\left(\mathbf{u}^{n}\right) \mathbf{u}^{n}+v D_{\varepsilon} \mathbf{u}^{n}-\frac{1}{\rho} G_{\varepsilon} \mathbf{p}^{n+1}+F_{\varepsilon}-\hat{R}_{\varepsilon} \mathbf{u}^{n+1}
\end{aligned}
$$

In (27), the subscripts $\varepsilon$ indicate that we have accounted for the porosity in the space discretization. Note that the convective term is non-linear, i.e. the coefficients of the matrix depend on the velocity $u$. Also note in this equation that the convective and viscous term are explicit and the pressure term and the friction force term are implicit.

We introduce an auxiliary vector field $\tilde{\mathbf{u}}^{n}$ :

$$
\tilde{\mathbf{u}}^{n}=\mathbf{u}^{n}-\Delta t \Omega_{\varepsilon}^{-1}\left(C_{\varepsilon}\left(\mathbf{u}^{n}\right) \mathbf{u}^{n}-v D_{\varepsilon} \mathbf{u}^{n}-F_{\varepsilon}\right)
$$

combine it with the momentum equation and rearrange terms to obtain:

$$
\mathbf{u}^{n+1}=\left[1+\Delta t \Omega_{\varepsilon}^{-1} \hat{R}_{\varepsilon}\right]^{-1}\left(\tilde{\mathbf{u}}^{n}-\frac{\Delta t}{\rho} \Omega_{\varepsilon}^{-1} G_{\varepsilon} \mathbf{p}^{n+1}\right)
$$

Eq. (29) is substituted into the discrete continuity equation. The result is a Poisson equation for the pressure that reads:

$$
M_{\varepsilon}\left[1+\Delta t \Omega_{\varepsilon}^{-1} \hat{R}_{\varepsilon}\right]^{-1} \Omega_{\varepsilon}^{-1} G_{\varepsilon} \mathbf{p}^{n+1}=\frac{\rho}{\Delta t} M_{\varepsilon}\left[1+\Delta t \Omega_{\varepsilon}^{-1} \hat{R}_{\varepsilon}\right]^{-1} \tilde{\mathbf{u}}^{n}
$$


From this equation the pressure is solved, after which the velocities at the new time level are found from (29).

\section{SETUP OF THE PHYSICAL MODEL TESTS}

Figure 4 shows the breakwater roundhead in the Scheldt Flume and the locations of the wave gauges. The breakwater roundhead has a diameter of $2 \mathrm{~m}$ at the base of the structure. The slope of the structure is $1: 1.5$ and the crest is at $0.5 \mathrm{~m}$ above the bottom. At the crest, the diameter of the roundhead is around $0.25 \mathrm{~m}$. The numbers in red in Figure 4 are used to identify the wave gauges when numerical results are compared to experimental results. A total of 18 wave gauges was placed in the flume: 15 were placed around the structure, 3 were positioned inside the structure. The 3 wave gauges inside the structure were placed in highly permeable PVC tubes. The wave gauges in the PVC tubes were found to be linear over the distance inside the structure. There is a slight jump in the calibration factor at the transition from inside the structure to outside. Above the structure the wave gauges are linear again. The jump needs to be accounted for in the processing of the measurement results. In the tests discussed in this paper, however, no overtopping occurred and no correction of the results was required
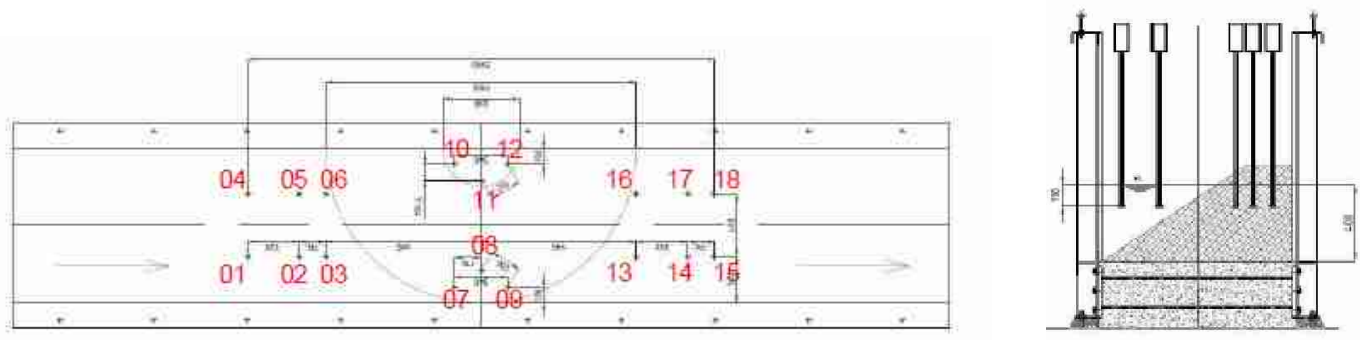

Figure 4. Plan view and side view of the breakwater roundhead in the Scheldt Flume at Deltares. Three wave gauges are placed inside the structure, 15 were placed around. The numbers in red uniquely identify the wave gauges.

The structure is composed of rock of a single grading, $D_{85} / D_{15}=1.43$. The average unit diameter $D_{n 50}$ for this material is $0.036 \mathrm{~m}$. This value was used to derive the coefficients for the numerical simulations, see Van Gent (1995). The porosity of the structure is $n=0.44$. The rocks near the free surface were grouted together with a marginal amount of adhesive material, just enough to prevent damage to the structure from occurring. Figure 5 shows what the structure looked like after construction. There is also one photo in Figure 5 that shows that tests were performed without structure. This will be discussed below.
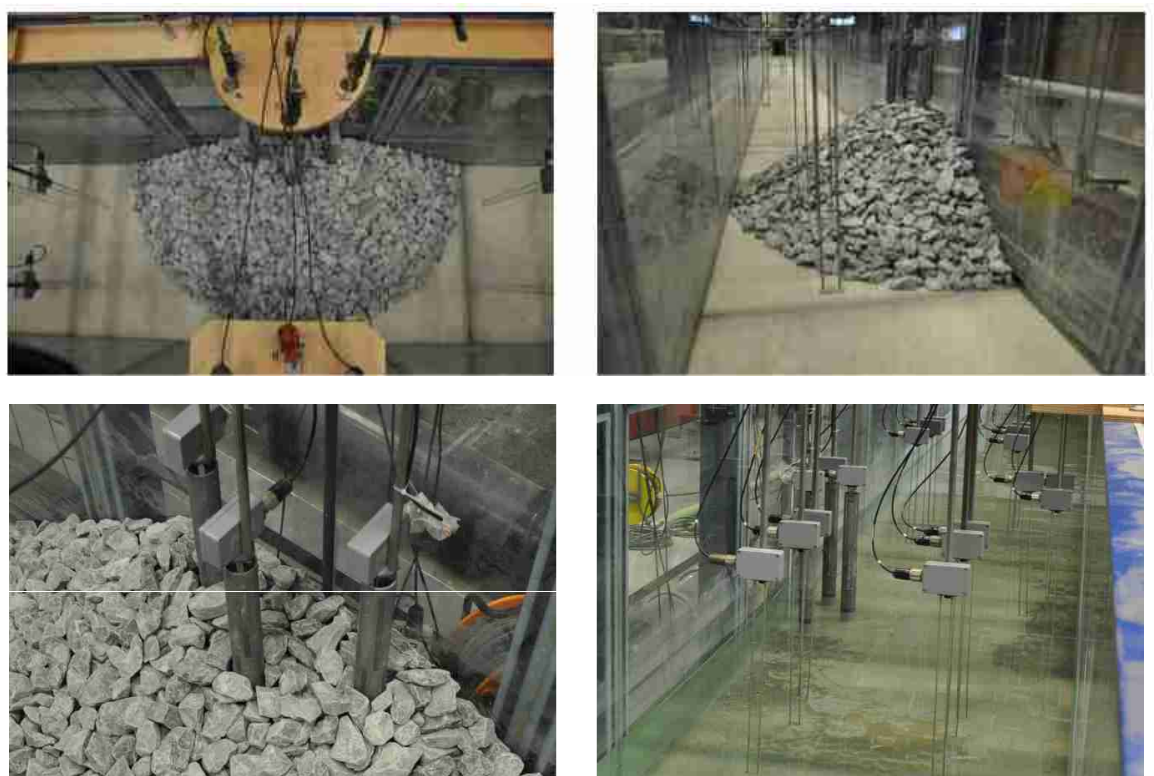

Figure 5. Breakwater roundhead in the flume after construction. Bottom right: undisturbed wave tests without structure but with instruments at the same positions as in the tests with structure. 
Two sets of 81 tests are performed. The first set of tests includes the structure in the flume. Waves were generated at the wave board end of the flume; at the other end, a spending beach prevents spurious reflection of waves back towards the structure. Tests were performed with regular (with period $T$ and wave height $H$ ) and irregular waves (with peak period $T_{p}$ and wave height $H_{m 0}$ ). Three base periods were chosen, corresponding to wavelengths that agree with $0.5 d_{b}, d_{b}$ and $2 d_{b}$, in which $d_{b}$ is the base diameter of the structure. For each of these base periods, the wave height is varied in such a way that the deep water wave steepness (based on $T$ or $T_{p}$ ) satisfies values between $1 \%$ and $5 \%$ in steps of $0.5 \%$. The water depth was kept constant at $h=0.40 \mathrm{~m}$ throughout the experiment. An overview of the test programme with structure is provided in Table 1.

\begin{tabular}{|c|c|c|c|c|c|}
\hline Number & Type & Length & Period & Steepness & Wave height \\
\hline 1 & \multirow{11}{*}{ Regular 5T Bursts } & \multirow{9}{*}{0.995} & \multirow{9}{*}{0.8} & 0.010 & 0.010 \\
\hline 2 & & & & 0.015 & 0.015 \\
\hline 3 & & & & 0.020 & 0.020 \\
\hline 4 & & & & 0.025 & 0.025 \\
\hline 5 & & & & 0.030 & 0.030 \\
\hline 6 & & & & 0.035 & 0.035 \\
\hline 7 & & & & 0.040 & 0.040 \\
\hline 8 & & & & 0.045 & 0.045 \\
\hline 9 & & & & 0.050 & 0.050 \\
\hline $10-18$ & & 1.990 & 1.2 & $0.010-0.050$ & $0.020-0.100$ \\
\hline $19-27$ & & 3.980 & 2.1 & $0.010-0.050$ & $0.040-0.200$ \\
\hline $28-36$ & \multirow{3}{*}{ Regular } & 0.995 & 0.8 & $0.010-0.050$ & $0.010-0.050$ \\
\hline $37-45$ & & 1.990 & 1.2 & $0.010-0.050$ & $0.020-0.100$ \\
\hline $46-54$ & & 3.980 & 2.1 & $0.010-0.050$ & $0.040-0.200$ \\
\hline $55-63$ & \multirow{3}{*}{ Irregular } & 0.995 & 0.8 & $0.010-0.050$ & $0.010-0.050$ \\
\hline $64-72$ & & 1.990 & 1.2 & $0.010-0.050$ & $0.020-0.100$ \\
\hline $73-81$ & & 3.980 & 2.1 & $0.010-0.050$ & $0.040-0.200$ \\
\hline
\end{tabular}

The second set of tests was performed with the same wave conditions but without the structure. Due to the 3D nature of the experiment, incoming waves are not straightforwardly separated from reflected (diffracted) waves originating from the structure. From the tests without structure, therefore, the incoming wave signal for the numerical simulations was determined. In all tests, measurements commenced before the wave board would start moving. 
Some of the tests with regular waves were performed with so-called wave bursts, in which we would keep measuring after the wave board had stopped. The 5 period long bursts were so short, that the entire wave train had passed the structure, before spurious reflection from the spending beach had time to reach the structure again. This is especially important for the longer period waves with small steepness, because the spending beach does not perform as well for these wave components as for the others. The wave bursts allow us investigate the results in high detail and to match the simulation results to the experimental results in their entirety. With the wave burst, we can separate model errors from other errors, such as errors at the inflow boundary or errors at the outflow boundary. For this reason, we focus on the wave bursts in this paper and leave the experiments with regular waves and irregular waves for a later study.

\section{SETUP OF THE SIMULATIONS}

The numerical simulations for the wave bursts are performed with ComFLOW. The computational domain is 3D. Figure 6 shows a snapshot of simulation R18, see Table 1. The numerical simulations are performed with a domain that covers the full width of the flume, $1 \mathrm{~m}$, but not the full length. The length of the domain in the direction of wave propagation is $6 \mathrm{~m}$, with the breakwater roundhead in the middle. The domain is also not defined over the full height of the flume, but only up to a level that is above the maximum expected free surface excursion. In vertical direction, the domain goes from $z=-0.4 \mathrm{~m}$ at the bottom to $z=0.3 \mathrm{~m}$, therefore $0.7 \mathrm{~m}$ in total. The outer dimensions of the breakwater in the computational domain are exactly the same as those of the experiment. The wave gauges were also placed at the same locations as in the experiment.

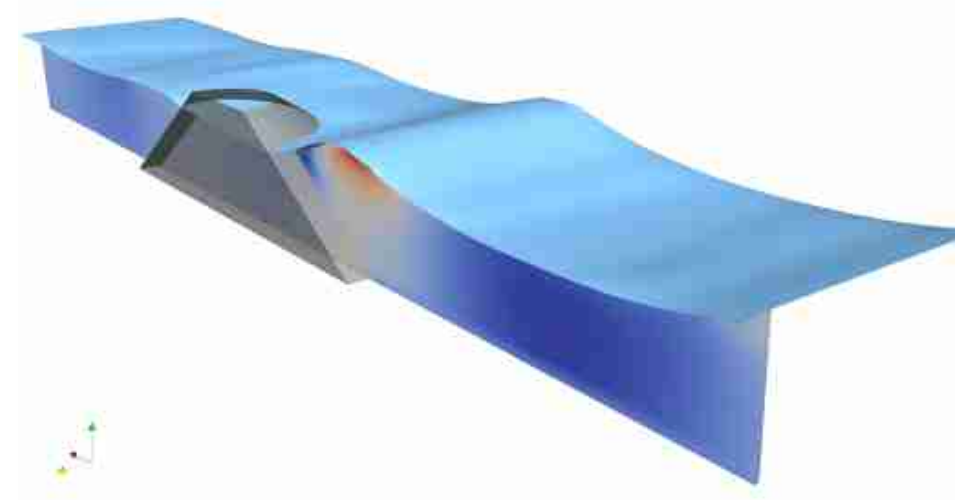

Figure 6. Snapshot of simulation R18, see Table 1.

The amplitudes and phases of the frequency components were determined from the free surface measurements at wave gauge WHM01, see Figure 4. Linear potential theory for waves was used to displace this information to the inflow boundary at $x=-3 \mathrm{~m}$. At the inflow end of the domain, a Generating Absorbing Boundary Condition (GABC) is specified, see Wellens et al. (2009). The same boundary condition was used at the outflow end of the domain, but no waves enter the domain at the outflow boundary. The side walls of the domain are closed just as the side walls of the Scheldt Flume.

In the $x$-direction, the direction of wave propagation, 150 cells were specified. The grid size in this direction then is $0.04 \mathrm{~m}$. 25 Cells were specified in $y$-direction, the grid size there then is the same as in the $x$-direction. It was found in previous studies that the accuracy of the free surface displacement algorithm depends on the aspect ratio of the cells near the free surface. The closer to 1 the aspect ratio is, the better the results. For this reason, also in vertical direction a grid size of $0.04 \mathrm{~m}$ was specified.

The time step is allowed to vary along with the CFL number. When it exceeds 0.9 the time step is halved. When the CFL number is smaller than 0.4 during 10 consecutive time steps, the time step is doubled. All simulations were for 65 seconds; in this time the wave bursts have entered and left the domain.

27 3D Simulations were performed in total, for all tests with wave bursts. In addition, 27 2D simulations were performed without the structure to compare the undisturbed wave signal in the 
simulations to the undisturbed wave signal in the experiment. In this way, we are able to separate model errors due to the interaction with the structure from errors due to boundary condition effects.

\section{COMPARISON BETWEEN SIMULATIONS AND EXPERIMENTAL RESULTS}

The first test that we will study is R01, see Table 1. Figure 7 shows the amplitude spectrum from the free surface registration at WHM01. What we can see is that the base frequency $(1.24 \mathrm{~Hz}$ corresponding to a wave period of $0.8 \mathrm{~s}$ ) is dominantly present. There is hardly any energy at the sum frequency position $(2.48 \mathrm{~Hz})$, indicating that non-linear effects are small in this experiment.

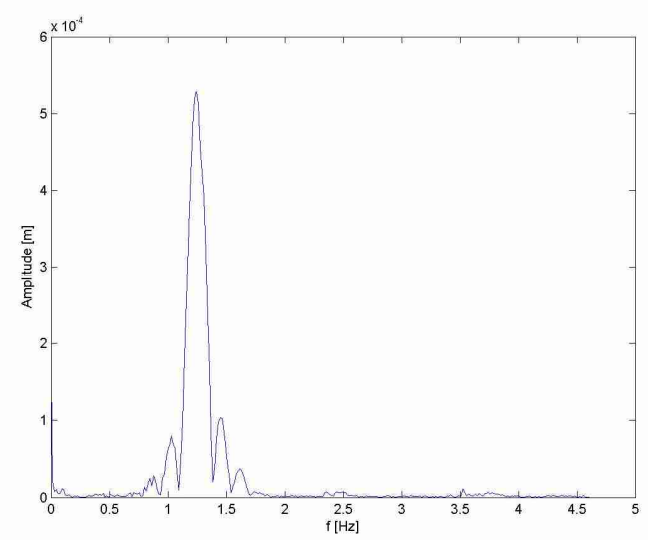

Figure 7. Amplitude spectrum of the free surface at WHM01 in test R01.

When non-linear effects are small, linear potential theory for waves, which is used to specify the inflow boundary conditions in terms of free surface and velocity, is a good model of the flow dynamics. This is apparent in the 2D simulation for the undisturbed waves in test R01, see Figure 8.

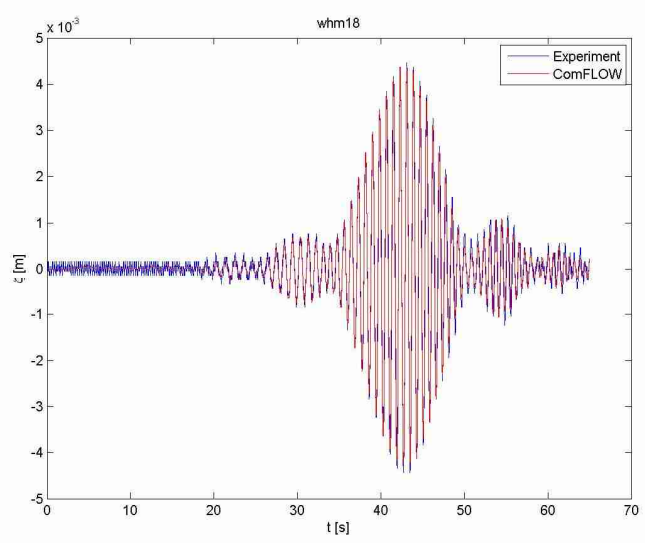

Figure 8. Free surface elevation at WHM18 in the 2D undisturbed wave simulation in ComFLOW compared to undisturbed wave test R01.

In Figure 8, the surface elevation at WHM18 from the 2D CoMFLOW simulations is compared to undisturbed wave test R01. The COMFLOW results are almost identical to the experimental results, from which we derive that the simulation results are an accurate representation of the flow dynamics in the flume for this wave condition.

However, when we look at Figure 9, in which the results of the 3D simulation with the structure are compared to test R01 with structure, there is more going on. The left plot of Figure 9 shows the 
phreatic surface at WHM10 inside the structure. The agreement of simulation and experiment is fair but not perfect. The same conclusion applies to the right plot of Figure 9, in which the free surface at WHM1 8 is shown. Here the simulation results are an overestimation of the experimental results. The simulation results could improve by calibrating the permeability coefficients. It should be noted that the permeability coefficients are a material property of the structure and can only be calibrated once. They should be the same for each of the experiments with this structure. There may also be a boundary condition effect if the direction of the diffracted waves is larger than 30 degrees with respect to the $x$-axis. This can be investigated by increasing the domain size at the outflow end and should be the subject of further research.
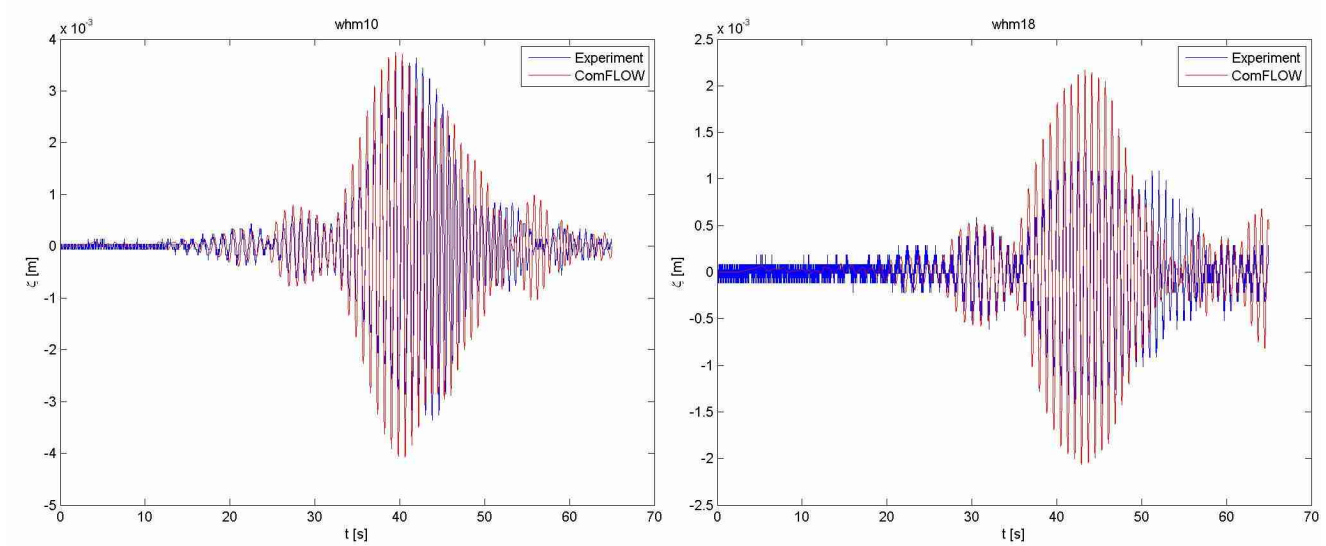

Figure 9. Surface elevation at WHM10 (left) and WHM18(right) in the 3D CoMFLOW simulation compared to test R01.

The next test that we will discuss is R09. Figure 10 shows the amplitude spectrum of the free surface elevation at WHM01 in undisturbed wave test R09. Test R09 has the same base frequency as test R01, but the specified wave height $H$ is 5 times as high. In this test, there is wave energy at the second harmonic $(2.48 \mathrm{~Hz})$.

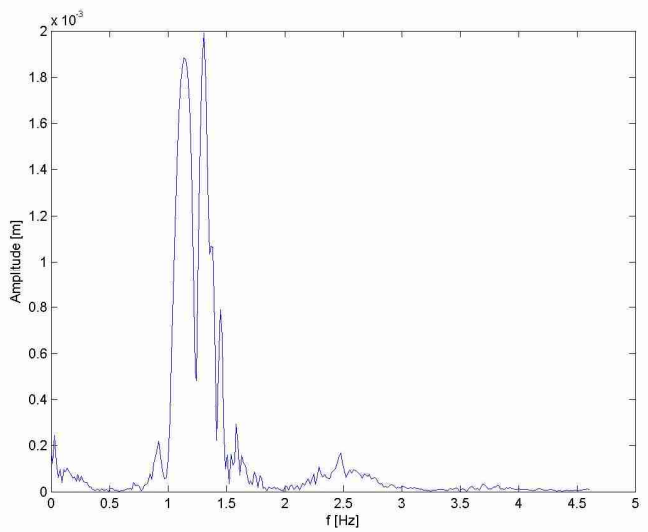

Figure 10. Amplitude spectrum of the free surface at WHM01 in test R09.

When the second harmonic contains wave energy, it is not certain that non-linear effects near the inflow boundary can be ignored. Linear theory may not be a good enough model to represent the flow dynamics at the inflow boundary of the numerical domain, as we will see in the next figure. 


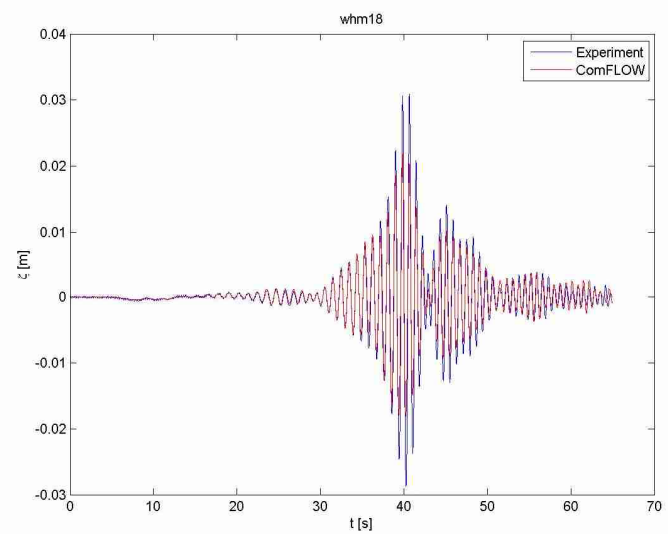

Figure 11. Free surface elevation at WHM18 in the 2D undisturbed wave simulation in ComFLOW compared to undisturbed wave test R09.

Figure 11 shows the surface elevation at WHM18 from the 2D undisturbed wave simulation compared to the undisturbed wave test R09. The simulation results still compare well to the experimental results, but the free surface in the simulation is no longer exactly identical to the experiment.

We believe this has to do with the approximation we have to make near the inflow boundary. Here, linear potential theory for waves used to specify the orbital velocities for each of the frequency components in the amplitude spectrum (Figure 10). The frequency components near the second harmonic at $2.48 \mathrm{~Hz}$ are specified as freely propagating waves, whereas we know from Stokes theory that the second harmonic is bound to the base frequency. This introduces an error. The error can be reduced by including second, or higher, order corrections in the signals at the boundary. Another way to reduce the error is to simulate the flow dynamics in the entire flume, starting at the wave board. Neither way to reduce the error is straightforward to accomplish. The second order corrections are difficult to derive. Simulating from the wave board is prohibitively expensive in terms of computational effort. Both measures should be the subject of further research.

If we take the errors near the inflow boundary for what they are and perform a 3D simulation that includes the structure, then the results are as shown in Figure 12.
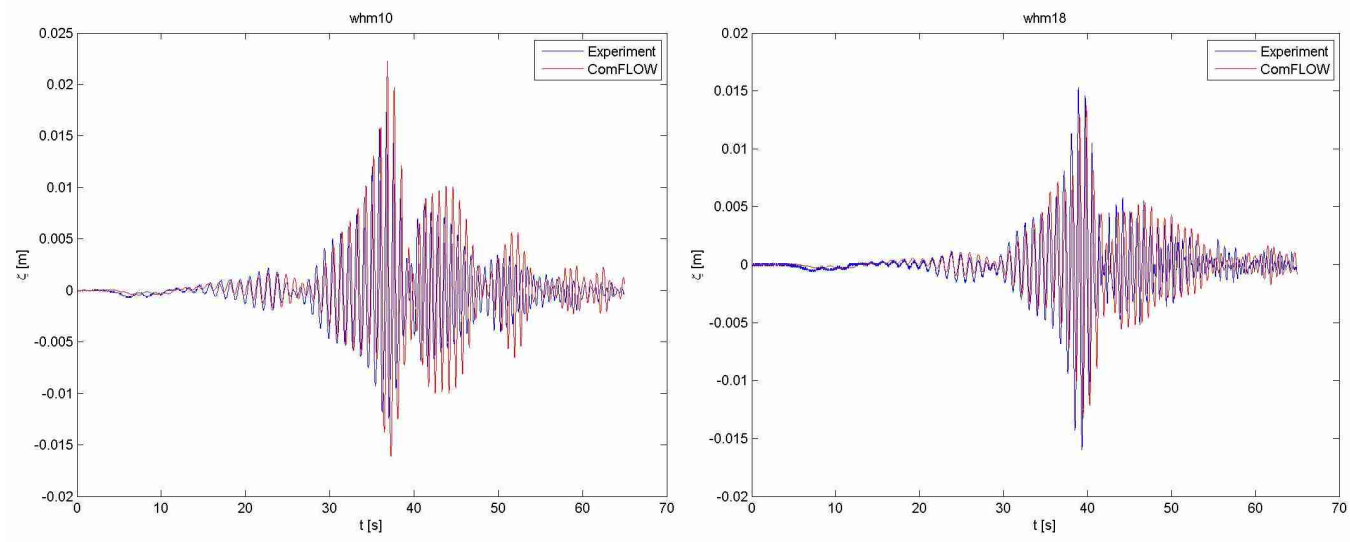

Figure 12. Surface elevation at WHM10 (left) and WHM18 (right) in the 3D COMFLOW simulation compared to test R09.

In Figure 12, the plot on the left shows the phreatic surface elevation at WHM10 inside the structure. The simulation results compare well to the measurements, but it should be possible to get better results by calibrating the permeability coefficients. The plot on the right in Figure 12 compares the free surface in the 3D simulation at WHM18 with the free surface in test R09. Simulation and experiment agree well. 
The next simulation that we will compare with the experiment is R19. The base frequency in R19 is lower than the tests that were discussed above, but the wave height over wave length ratio, or slope, in R19 is the same as in R01, indicating that a similar amount of non-linearity can be expected. Figure 13 shows the amplitude spectrum at WHM01 in experiment R19.

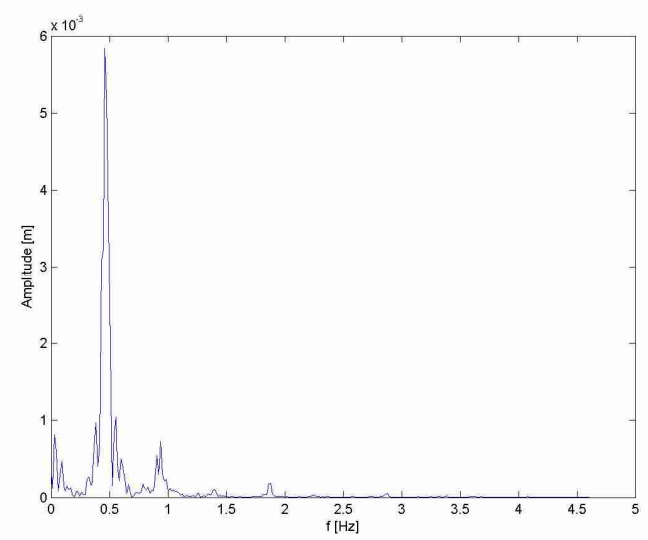

Figure 13. Amplitude spectrum of the free surface at WHM01 in test R19.

In Figure 13, the base frequency is equal to $0.47 \mathrm{~Hz}$. This is clearly the dominant frequency. But, whereas R01 in Figure 10 had hardly any wave energy at the second harmonic, test R19, with the same slope as R01, has a distinct secondary peak at $0.94 \mathrm{~Hz}$. Apparently, for a water depth of $0.4 \mathrm{~m}$, the slope is not the only measure of non-linearity. Further study may yield a dimensionless parameter that does allow us to compare the results between tests: possibly the wave height over depth ratio, or the Ursell number.

In any case, non-linearity cannot be ignored in test R19 and that will become apparent in the simulation results. Figure 14 shows the free surface at WHM18 in the 2D ComFLOW simulation compared to undisturbed wave test R19.

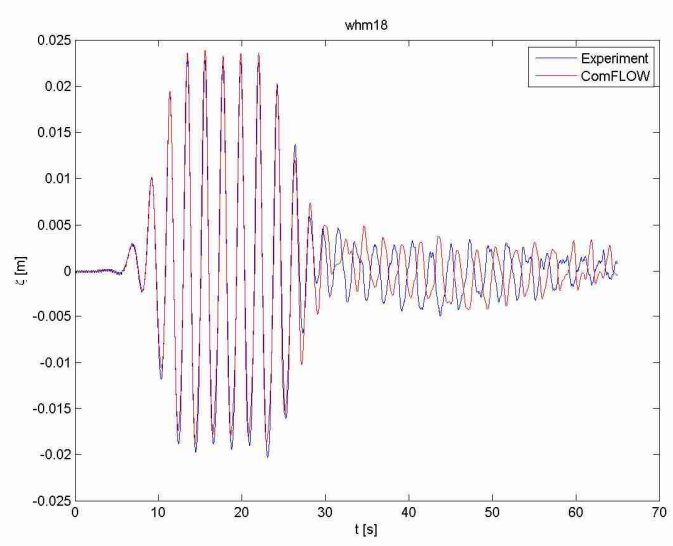

Figure 14. Free surface elevation at WHM18 in the 2D undisturbed wave simulation in ComFLOW compared to undisturbed wave test R19

In Figure 14, the free surface at WHM18 in 2D COMFLOW compares reasonably well to the experiment. At the start of the simulation, the COMFLOW result is identical to the experiment. However, when the wave burst has passed through the domain, there is a distinct difference in phase between the simulation result and the undisturbed wave experiment R19. The phase difference is almost certainly due to linearization errors in prescribing the incoming wave information.

The 3D simulation results are shown in Figure 15, where they are compared to test R19. The left plot in Figure 15 shows the phreatic surface inside the structure at WHM10. The right plot shows the 
free surface at WHM18. The phase difference after 40s between simulation and experiment is clearly visible in these simulations. The agreement between simulation and experiment R19, otherwise, is fair, although the phreatic surface excursion inside the breakwater roundhead is slightly underpredicted.
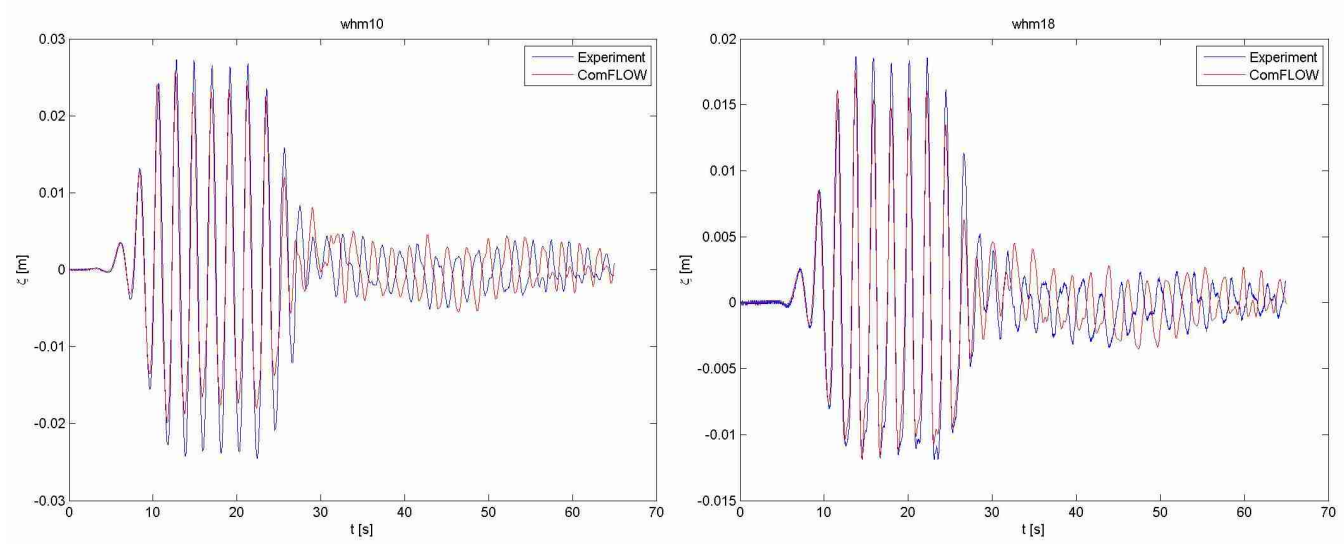

Figure 15. Surface elevation at WHM10 (left) and WHM18(right) in the 3D CoMFLOW simulation compared to test R19.

To conclude this section, in which simulation results are compared to the measurements, we will show a number of wave gauges from experiment R27 to demonstrate the overall performance of COMFLOW for the wave burst experiment with the highest waves. The surface elevations are shown in Figure 16. Some smaller details in Figure 16 do not compare, but the agreement between COMFLOW and the experiment, also inside the structure (top right in Figure 16) is good.
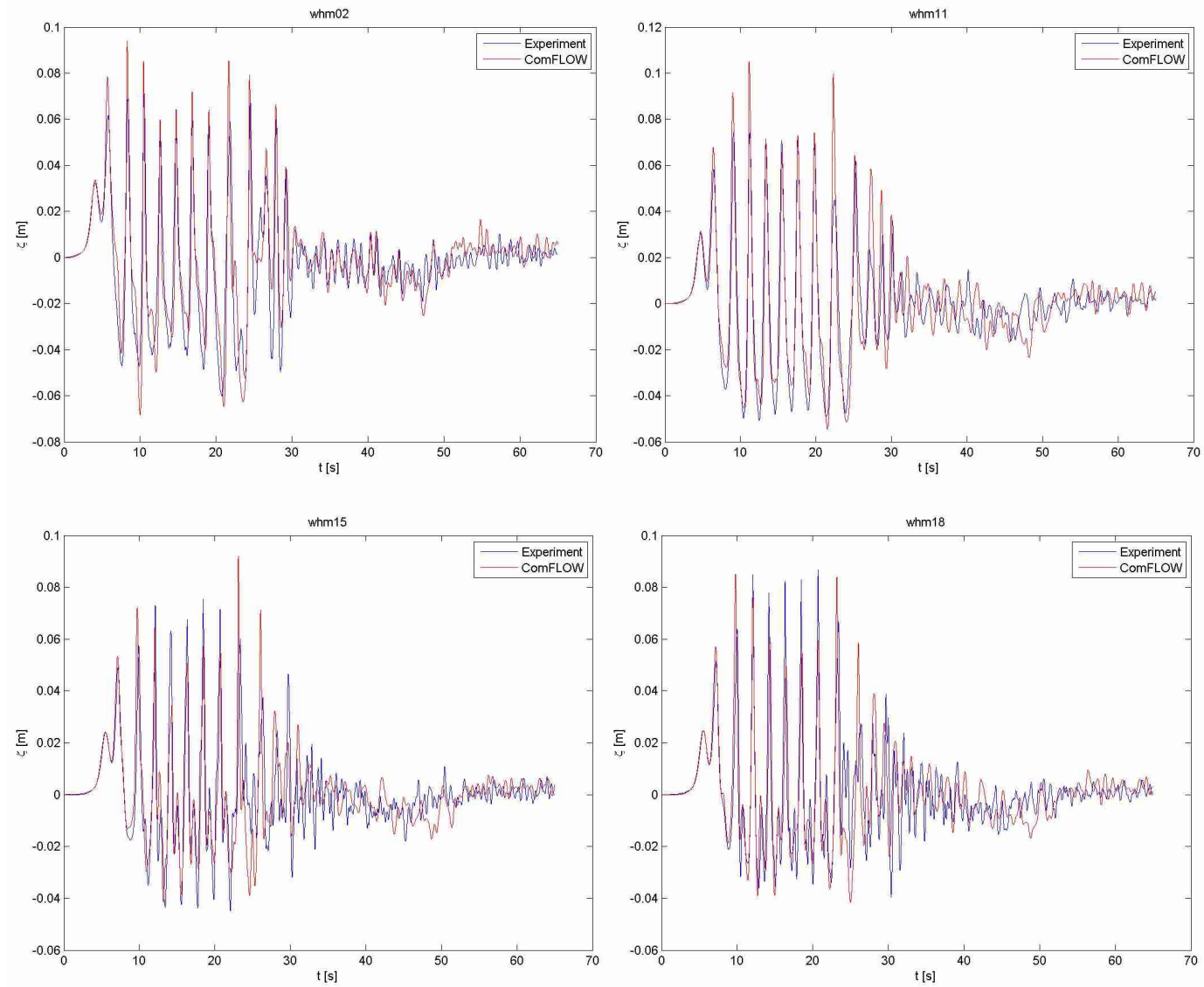

Figure 16. Surface elevation at WHM02 (top left), phreatic surface at WHM11 (top right), WHM15 (bottom left) and WHM18 (bottom right) in the 3D CoMFLOW simulation compared to test R27. 


\section{CONCLUSIONS}

COMFLOW, which is based on the 3D Navier-Stokes equations in porous media, has been validated in $3 \mathrm{D}$ with a tailor-made physical model test. The model tests were conducted with a breakwater roundhead in the Scheldt Flume. Although not an experiment that is correct for breakwater roundheads in general because of side-wall reflections, these side wall reflections and the overall complexity of the flow near the structure were an excellent validation opportunity for COMFLOW.

The physical model tests comprised 81 tests with structure and 81 undisturbed wave tests to determine the undisturbed incoming wave for COMFLOW. Some experiments were conducted with socalled wave bursts. This paper focuses only on these 27 experiments.

2D COMFLOW simulations were compared to the undisturbed wave tests. 3D ComFLOW simulations were compared to the tests with the structure in the flume. From the comparison we find:

1. COMFLOW is in good agreement with the measurements when the assumption of linearity near the inflow boundary is correct. When super- and subharmonics become important another method to prescribe waves at the inflow boundary should be used. Second order correction of the signals is one such method, but this has to be investigated in further research.

2. When super- and subharmonics are present in the wave signals, slight phase differences between COMFLOW results and the experiment can be observed.

3. In some tests, the agreement of the phreatic surface inside the structure between COMFLOW and the experiment could improve by calibration of the permeability coefficients. Note that these coefficients are a material property of the structure and that there is one set of coefficients that is the same for all tests.

4. The overall performance of COMFLOW for wave motion near permeable structures in 3D is good.

\section{REFERENCES}

Honda, T., P.R.Wellens and M.R.A. van Gent. 2011. Numerical Simulations for Wave Overtopping by Irregular Waves, $5^{\text {th }}$ International Conference on Advanced Computational Methods in Engineering, Liege, Belgium

Kleefsman, K.M.T., G. Fekken, A.E.P. Veldman, B. Iwanowski, and B. Buchner. 2005. A Volume-ofFluid based simulation method for wave impact problems, Journal of Computational Physics, 206(1): 363-393.

Rienecker, M.M. and J.D. Fenton. 1981. A Fourier Approximation Method For Steady Water-Waves, Journal of Fluid Mechanics, 104(MAR):119-137, 1981.

Seelig, W.N. and J.P. Ahrens. 1981. Estimation of wave reflection and energy dissipation coefficients for beaches, revetments and breakwaters, TP81-1, CERC, US Army Corps of Engrs, Vicksburg, MS.

Van Gent, M.R.A. 1995. Porous flow through rubble mound material, J. of Waterway, Port, Coastal and Ocean Engineering, Vol.121, no.3, pp.176-181, ASCE, New York.

Verstappen, R.W.C.P. and A. E. P. Veldman. 2003. Symmetry-preserving discretization of turbulent flow, Journal of Computational Physics, 187(1):343-368.

Wellens, P.R., R. Luppes, A.E.P. Veldman and M.J.A. Borsboom. 2009. CFD Simulations of a SemiSubmersible with Absorbing Boundary Conditions, Proceedings of OMAE 2009, Honolulu.

Wellens, P.R., M.J.A. Borsboom and M.R.A. van Gent. 2010. 3D simulation of wave interaction with permeable structures, Proceedings of $32^{\text {nd }}$ International Conference on Coastal Engineering, Shanghai, China.

Wenneker, I., P.R.Wellens and R. Gervelas. 2010. Volume-of-Fluid model CoMFLOW simulations of wave impacts on a dike, Proceedings of $32^{\text {nd }}$ International Conference on Coastal Engineering, Shanghai, China. 